\title{
Pretreatment with human urine-derived stem cells protects neurological function in rats following cardiopulmonary resuscitation after cardiac arrest
}

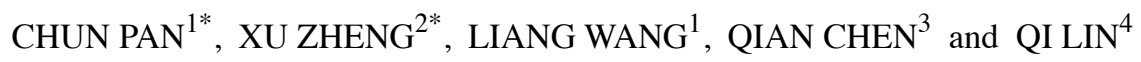 \\ ${ }^{1}$ Emergency Department, Suzhou Emergency Center; ${ }^{2}$ Department of Anesthesiology, \\ The Affiliated Suzhou Science and Technology Town Hospital of Nanjing Medical University; \\ ${ }^{3}$ Laboratory Center, The Affiliated Suzhou Hospital of Nanjing Medical University, Suzhou, Jiangsu 215008; \\ ${ }^{4}$ Dispatch Department, Suzhou Emergency Center, Suzhou, Jiangsu 215000, P.R. China
}

Received November 29, 2019; Accepted July 10, 2020

DOI: $10.3892 /$ etm.2020.9240

\begin{abstract}
Cardiopulmonary resuscitation (CPR) after cardiac arrest (CA) often leads to neurological deficits in the absence of effective treatment. The aim of the present basic research study was to investigate the effects of human urine-derived stem cells (hUSCs) on the recovery of neurological function in rats after CA/CPR. hUSCs were isolated in vitro and identified using flow cytometry. A rat model of CA was established, and CPR was performed. Animals were scored for neurofunctional deficits following hUSC transplantation. The expression levels of brain-derived neurotrophic factor (BDNF) and vascular endothelial growth factor (VEGF) in the hippocampus and temporal cortex were detected via immunofluorescence. Moreover, brain water content and serum S100 calcium binding protein B (S100B) levels were measured 7 days following hUSC transplantation. The results demonstrated that hUSCs had upregulated expression levels of CD29, CD90, CD44, CD105, CD73, CD224 and CD146, and expressed low levels of CD34 and human leukocyte antigen-DR isotype. In addition, hUSCs were able to differentiate into neuronal cells in vitro. The SPSS 19.0 statistical package was used for statistical analysis, and it was found that the neurological function of the rats after $\mathrm{CA} / \mathrm{CPR}$ was significantly improved following
\end{abstract}

Correspondence to: Dr Qi Lin, Dispatch Department, Suzhou Emergency Center, 89 Xujiang Road, Suzhou, Jiangsu 215000, P.R. China

E-mail: 18914037985@189.cn

Dr Qian Chen, Laboratory Center, The Affiliated Suzhou Hospital of Nanjing Medical University, 242 Guangji Road, Suzhou, Jiangsu 215008, P.R. China

E-mail: sdfeycq@sina.com

*Contributed equally

Key words: human urine-derived stem cells, neurological function, cardiopulmonary resuscitation, rats
hUSC transplantation. Furthermore, hUSCs aggregated in the hippocampus and temporal cortex, and secreted large amounts of BDNF and VEGF. hUSC transplantation also effectively inhibited brain edema and serum S100B levels after CPR. Therefore, the results suggested that hUSC transplantation significantly improved the neurological function of rats after $\mathrm{CA} / \mathrm{CPR}$, possibly by promoting the expression levels of BDNF and VEGF, as well as inhibiting brain edema.

\section{Introduction}

Cardiac arrest (CA) for $>5$ min often leads to irreversible brain damage (1). Implementing effective brain resuscitation to save ischemic neurons and accelerate the repair of damaged nerve tissue to maintain normal nerve function has become a global challenge $(2,3)$, but the study of drug-induced cerebral resuscitation has progressed slowly $(4,5)$. Although hypothermia therapy has certain neuroprotective effects, it remains difficult to reverse neurological damage following $\mathrm{CA} /$ cardiopulmonary resuscitation (CPR) (6). Currently, there is no effective treatment for improving neurological function after CA/CPR, although numerous clinical and basic studies have been performed to identify effective therapies to improve the quality of cerebral resuscitation (7).

Oxidative stress injury serves a key role in cerebral ischemia. The abrupt cessation of blood flow often leads to cerebral ischemia and hypoxia followed by CA, and ATP, an oxygen-dependent membrane ion transporter, stops functioning when ischemia and hypoxia occur (8). Subsequently, calcium floods into cells and promotes the apoptosis of brain cells (8). In addition to excitotoxicity, the excessive stimulation of neurotransmitters can also lead to neuronal damage (9). Moreover, reperfusion injury following CPR may induce oxidative stress and promote the formation of free radicals and reactive oxygen species (ROS), which can react with numerous macromolecules and destroy intracellular macromolecules, including DNA, proteins and lipids $(10,11)$.

Human urine-derived stem cells (hUSCs) have been reported to be promising candidates for tissue engineering therapies due to their expansion efficiency and multilineage 
differentiation properties, as well as their capacity to secrete vasoactive peptides that regulate the function of vessels (12). The advantages of the transplantation of hUSCs, which have become an ideal adult stem cell system, include the non-invasiveness of their collection and their wide range of sources (13). For example, hUSCs can be harvested from voided urine through a simple, and low-cost procedure. Additionally, hUSCs have been revealed to be involved in the repair and reconstruction of bone, skin, the intestinal tract and the urinary tract (12). Previous studies have shown that the transplantation of hUSCs can improve the function of the kidney and bladder in pathological model rats $(14,15)$. Another study revealed that hUSCs can promote vascular endothelial growth factor (VEGF) expression in collagen hydrogels during myogenesis and innervation following subcutaneous implantation in nude mice (16). In addition, hUSC transplantation is beneficial for wound recovery in diabetic patients (17). Therefore, hUSCs are considered promising multipotent stem cells.

To the best of our knowledge, no studies of the protective effects of hUSCs on neurological function after CA/CPR have been previously reported. Thus, the aim of the present study was to investigate the effects of hUSCs on the recovery of neurological function in rats after CA/CPR. The current study evaluated the hypothesis that hUSC transplantation can effectively improve the neurological function of rats following $\mathrm{CA} / \mathrm{CPR}$, which may benefit patients with $\mathrm{CA}$ in the future.

\section{Materials and methods}

Ethical approval. This study was approved by the Ethics Committee of The Affiliated Suzhou Hospital of Nanjing Medical University. All urine donors provided informed written consent before giving urine samples. Voided urine samples $(80-400 \mathrm{ml})$ from four healthy men (age, 25-33 years) were collected between June and December 2018 at The Affiliated Suzhou Hospital of Nanjing Medical University. All animal experiments were conducted according to the National Institutes of Health guidelines (18).

Cell isolation, culture and identification. Volumes of $300 \mathrm{ml}$ urine, either pooled from multiple donors or from a single donor if the sample volume was low, were centrifuged at $500 \mathrm{xg}$ for $5 \mathrm{~min}$ at room temperature and then at 2,000 $\mathrm{x}$ g for $10 \mathrm{~min}$ at $4^{\circ} \mathrm{C}$. The cell pellets were then suspended in mixed medium composed of embryo fibroblast medium (EFM; Gibco; Thermo Fisher Scientific, Inc.) and keratinocyte serum-free medium (KSFM; Gibco; Thermo Fisher Scientific, Inc.; EFM-KSFM, 1:1 ratio) supplemented with 10\% FBS (Gibco; Thermo Fisher Scientific, Inc.). The cells were cultured at $37^{\circ} \mathrm{C}$ in a humidified atmosphere with $5 \% \mathrm{CO}_{2}$ in 24 -well plates $\left(\sim 10^{5} /\right.$ well) for 3-5 days, at which point hUSC clones appeared. When the cells reached $60-70 \%$ confluence, they were transferred to 6-well plates. The cells adhered to the bottom of the flask, and cell colonies formed (passage 1). At passage , hUSCs were seeded in a 6-well tissue culture plate at a density of $10^{3}$ cells $/ \mathrm{cm}^{2}$. The majority of these cells were adherent to the plate, and they displayed a mixture of morphologies.

The neuronal differentiation potential of hUSCs was evaluated using immunofluorescence staining for neurofilament protein-200 (NF200) and glial fibrillary acidic protein (GFAP) on day 14 . Briefly, cells $\left(5 \times 10^{4}\right)$ were seeded in 24 -well plates and washed with PBS. Cells were then fixed with $4 \%$ paraformaldehyde for $20 \mathrm{~min}$ at room temperature and blocked with $0.3 \%$ Triton X-100 in $0.5 \%$ BSA (Beyotime Institute of Biotechnology) for $1 \mathrm{~h}$. Primary antibodies for NF200 (cat. no. sc-32729; 1:300; Santa Cruz Biotechnologies Inc.), GFAP (cat. no. sc-33673; 1:500; Santa Cruz Biotechnologies Inc.) were added and incubated overnight at $4^{\circ} \mathrm{C}$. The Alexa Fluor ${ }^{\circledR} 488$-labeled secondary IgG antibody (cat. no. ab150157; 1:200; Abcam) was added for $3 \mathrm{~h}$ at $4^{\circ} \mathrm{C}$, and the cells were then incubated with DAPI (Beyotime Institute of Biotechnology) for a further $45 \mathrm{~min}$ at $4^{\circ} \mathrm{C}$. Cells were observed using a fluorescence microscope (Carl Zeiss AG). The expression levels of surface marker proteins, including CD29 (cat. no. ab134179), CD90 (cat. no. ab23894), CD44 (cat. no. ab6124), CD105 (cat. no. ab44967), CD73 (cat. no. ab202122), CD224 (cat. no. ab55138), CD146 (cat. no. ab75769), CD34 (cat. no. ab81289) and human leukocyte antigen-DR isotype (HLA-DR; cat. no. ab92511), on hUSCs were detected using flow cytometry (Beckman Coulter, Inc.). All flow cytometry antibodies were obtained from Abcam and the dilution of all antibodies was 1:100.

Experimental groups. Male Sprague-Dawley rats were purchased from Zhao Yan New Drug Research Center. Rats were housed in microisolator cages with sterile bedding and water and food was available ad libitum. The rats were maintained in an environment of $24-26^{\circ} \mathrm{C}$, at a humidity of $50-60 \%$ on $12 \mathrm{~h}$ light/dark cycles. Healthy male specific pathogen free Sprague-Dawley rats $(\mathrm{n}=36$; age, 6-12 weeks; weight, 200-300 g) were randomly divided into three groups: Sham (no CA/CPR model established; $n=12$ ), blank (CA/CPR model established; $n=12)$ and hUSCs (CA/CPR model established and cells added; $n=12$ ) groups. In the hUSCs group, $5 \times 10^{6} / 0.1 \mathrm{ml}$ hUSCs labeled with PKH26 (Merck KGaA) at room temperature for $3 \mathrm{~min}$, according to the manufacturer's instructions, were administered three times (with an interval of $6 \mathrm{~h}$ between each administration) via caudal vein injection 1 day before the CA/CPR model was established. Equivalent volumes of PBS to the volumes of cells were injected into the sham group and blank group (Fig. 1). Prior to injection the rats were anesthetized using pentobarbital sodium via intraperitoneal injection at a dose of $40 \mathrm{mg} / \mathrm{kg}$. The animals were allowed to recover from anesthesia and returned to their cages for 7 days.

Rat model of CA/CPR. The rat CA/CPR model were established based on previously published studies $(19,20)$. Rats were anesthetized using pentobarbital sodium via intraperitoneal injection at a dose of $40 \mathrm{mg} / \mathrm{kg}$. The rats were then fixed on the operating table in the supine position, and limb leads were connected for electrocardiographic monitoring. The $24 \mathrm{G}$ venous catheters were placed in the right femoral artery and left femoral vein, which were connected to the Powerlab system (ADInstuments Pty Ltd.), used to monitor blood pressure, and an infusion device (RWD Life Science Co., Ltd.), respectively. A $16 \mathrm{G}$ venous indwelling soft cannula was inserted via the mouth and connected to a small animal ventilator (RWD Life Science Co., Ltd.) for mechanical ventilation. The respiratory rate was adjusted to 80 times/min, the tidal volume was $6 \mathrm{ml} / \mathrm{kg}$ and the fraction of inspired oxygen was $21 \%$, and the parameters were kept stable for $10 \mathrm{~min}$. Changes in blood pressure and electrocardiographic changes were 


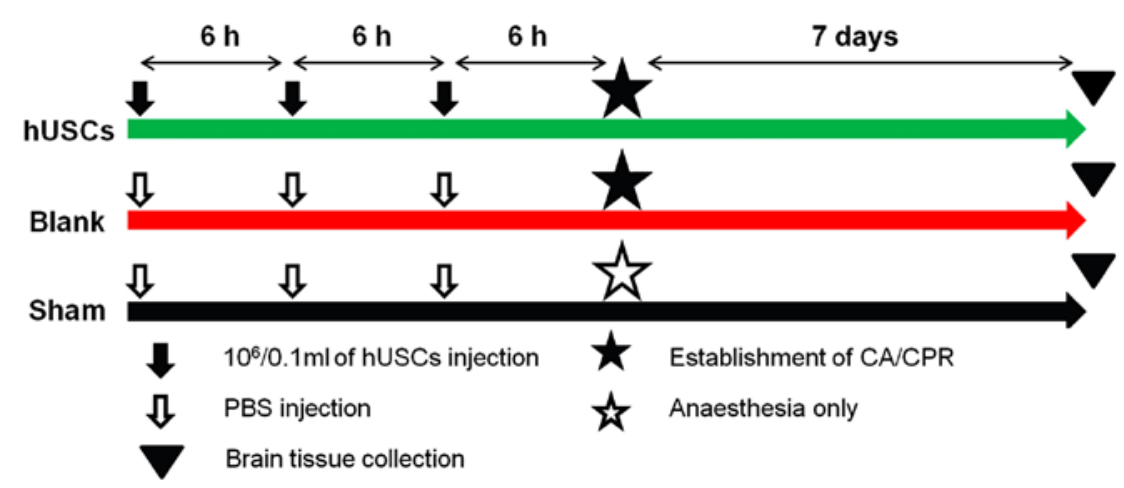

Figure 1. Schematic diagram of hUSC transplantation. hUSC were administered before the CA/CPR model was established. Equivalent volumes of PBS were injected into the sham group and blank group. hUSC, human urine-derived stem cells; CA/CPR, cardiac arrest/cardiopulmonary resuscitation.

closely observed after the clipping of the tracheal tube at the end of expiration. Systolic blood pressure (SBP) $<25 \mathrm{mmHg}$ was used as the criterion for CA.

Chest compression was performed at 200 times per min according to the rhythm of a metronome. The compression depth was maintained at $1 / 3$ of the diameter of the thorax of the rats. Adrenaline $(0.04 \mathrm{mg} / \mathrm{kg}$; Shanghai Harvest Pharmaceutical, Co., Ltd.) and $1 \mathrm{ml}$ Wanwen (hydroxyethyl starch 130/0.4 sodium chloride injection; cat. no. H2012043 Fresenius Kabi Deutschland $\mathrm{GmbH}$ ) were injected via the femoral vein, and then blood pressure and heart rate were measured, and electrocardiograms were recorded. Restoration of spontaneous circulation was defined as an increase in mean arterial pressure $>60 \mathrm{mmHg}$ lasting $\geq 10$ min following spontaneous rhythm (21). If spontaneous rhythm did not appear after 3 min of chest compressions or if the SBP fell $<60 \mathrm{mmHg}$, rescue was abandoned, and resuscitation was considered unsuccessful. Surviving rats continued to receive ventilator support, and mechanical ventilation was stopped after spontaneous breathing recovered.

During the operation, rectal temperature was monitored using a temperature feedback system and maintained at $37.0 \pm 0.5^{\circ} \mathrm{C}$. All rats were subcutaneously injected with $2 \mathrm{ml}$ $10 \%$ glucose every $6 \mathrm{~h}$ after the operation until the animals could drink water and eat independently, and the rats were placed in a separate cage with food at a constant temperature of $23^{\circ} \mathrm{C}$. The rats were euthanized using cervical dislocation at 7 days after surgery under deep anesthesia. No signs of pain or distress were observed throughout the whole process. Mortality was confirmed by physical signs of apnea, CA and absence of brain stem reflexes.

Immunofluorescence histochemistry. The hippocampus and temporal cortex were removed quickly after the rats were euthanized. Each region was identified and punched under a stereomicroscope. The hippocampus and temporal cortex were dissected from brain slices. Immunofluorescence histochemistry was performed as previously described (22). Briefly, after perfusion with 4\% PFA at room temperature overnight, dehydration through graded sucrose (20 and 30\%) and embedding in optimal cutting temperature compound (Sakura Finetek USA, Inc.), the tissue was cut at $5 \mu \mathrm{m}$ by freezing microtome (model, CM 1850; Leica Microsystems $\mathrm{GmbH}$ ) for immunofluorescence staining. Transverse spinal sections were cut using a cryostat and collected in 0.01 M PBS, pH 7.3. The tissues were incubated with $0.3 \%$ Triton $\mathrm{X}-100$ at room temperature for $30 \mathrm{~min}$ and then with rabbit anti-rat NF200 (cat. no. sc-32729; 1:300), GFAP (cat. no. sc-33673; 1:500), brain-derived neurotrophic factor (BDNF; cat. no. sc-65514; 1:500) and VEGF (cat. no. sc-7269; 1:200) primary antibodies (all from Santa Cruz Biotechnology, Inc.) overnight at $4^{\circ} \mathrm{C}$. Then, the slices were washed twice with PBS and incubated with secondary antibodies (rabbit anti-goat IgG; cat. no. ZF-0314; 1:100; Zhongshanjinqiao, Inc.) for $60 \mathrm{~min}$ at room temperature. The slices were observed under a fluorescent microscope (Carl Zeiss AG).

Analysis of brain water content. Resected brain tissues were wiped clean with filter paper before being weighed, to obtain the wet weight. The dry-wet ratio was used to analyze brain edema. The brain tissues were dried at $70^{\circ} \mathrm{C}$ overnight to determine the dry weight. Brain water content $(\%)=($ wet weight-dry weight)/wet weight $\mathrm{x} 100 \%$.

Serum $\mathrm{S100}$ calcium binding protein B (S100B) analysis. In total, $1 \mathrm{ml}$ fresh blood was extracted from the tail vein of each rat. The fresh blood was centrifuged at $1,000 \mathrm{x} \mathrm{g}$ for $10 \mathrm{~min}$ at room temperature after heparin anticoagulation, and the supernatant collected as serum for follow-up experiments. According to the instructions of a commercial ELISA kit (cat. no. ab234573; Abcam) the absorbance (optical density) was measured at $450 \mathrm{~nm}$ using a microplate reader, and the concentration of S100B was calculated according to a standard curve.

Western blotting. The total protein expression of cleaved caspase-3 (C-caspase-3; 1:1,000; cat. no. 9661s; Cell Signaling Technology, Inc.), Bax (1:1,000; cat. no. 2772s; Cell Signaling Technology, Inc.) and B-cell lymphoma 2 (Bcl-2; 1:1,000; cat. no. 59348; Abcam) and GAPDH (1:1,000; cat. no. AF1186; Beyotime Institute of Biotechnology) in tumor tissues was analyzed by western blotting. Western blotting was performed as previously described (23). Briefly, after quantification with a BCA kit (cat. no. P0012S, Beyotime Institute of Biotechnology), $20 \mu \mathrm{g}$ of protein samples were resolved by $10 \%$ SDS-PAGE and transferred to nitrocellulose membranes. The nitrocellulose membrane was then blocked with $5 \%$ nonfat milk for $1 \mathrm{~h}$ at room temperature. After washing, the nitrocellulose membrane was incubated with C-caspase-3 (1:1,000; cat. no. 9661s; Cell Signaling Technology), Bax (1:1,000; cat. no. 2772s; Cell Signaling Technology), BCL-2 (1:1,000; 
A
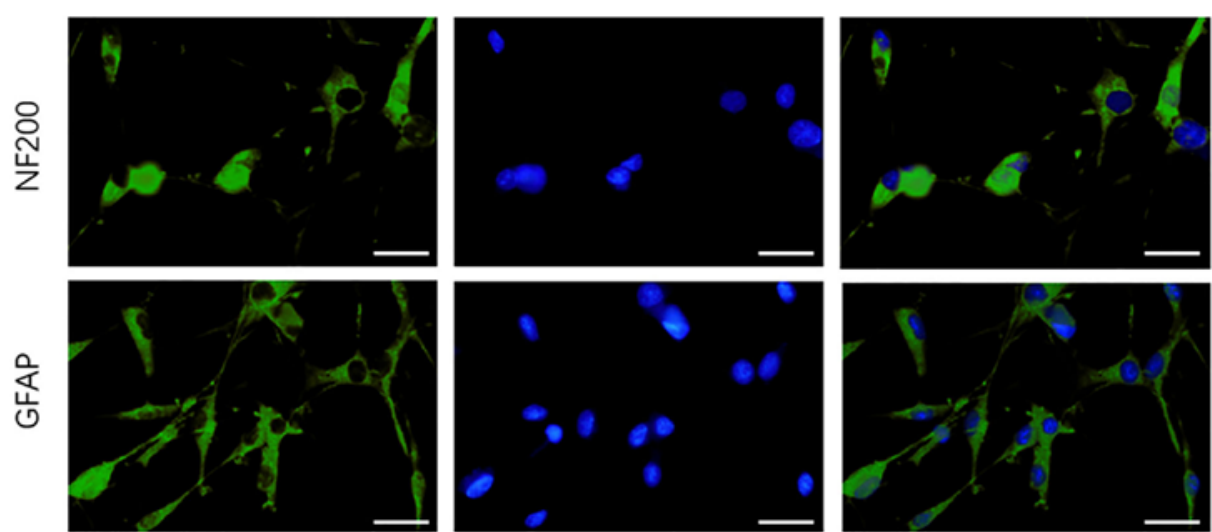

$\mathbf{B}_{100}$
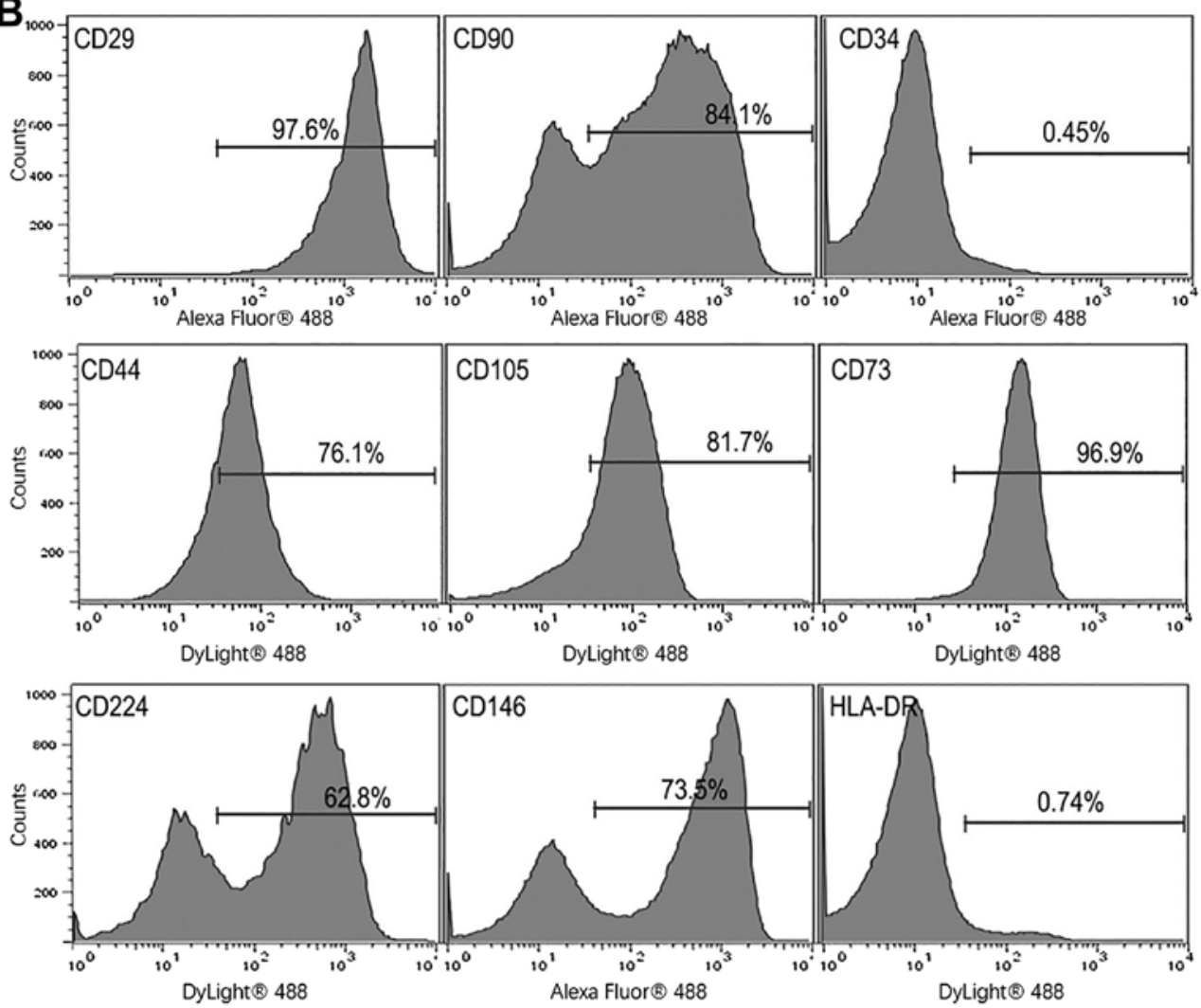

Figure 2. Identification of hUSCs. (A) NF200 and GFAP immunofluorescence of hUSC. Magnification, x100; scale bar, $50 \mu \mathrm{m}$. Increased protein expression levels of NF200 and GFAP were observed after neurogenesis induction. (B) Phenotypic expression of hUSCs was evaluated using flow cytometry. hUSCs had upregulated CD29, CD90, CD44, CD105, CD73, CD224 and CD146, and expressed low levels CD34 and HLA-DR. hUSC, human urine-derived stem cells; HLA-DR, human leukocyte antigen-DR isotype; GFAP, glial fibrillary acidic protein; NF200, neurofilament protein-200.

cat. no. 59348, Abcam, Cambridge, UK) and GAPDH antibody (1:1,000; cat. no. AF1186; Beyotime Institute of Biotechnology, China) overnight at $4^{\circ} \mathrm{C}$. After incubation with the secondary antibody (1:2,000; cat. no. A0181; Beyotime Institute of Biotechnology) for $2 \mathrm{~h}$ at room temperature, the membranes were then visualized using an ECL kit (Tanon Science and Technology Co., Ltd.) on the Tanon Imaging System.

Neural deficit scores (NDS). Neurological functions were assessed by three investigators blinded to the treatment using an established NDS scale (24), which evaluated general behavior, neurological function, sensory function, motor function and coordination. NDS of the surviving rats was assessed at days 1, 3 and 7 after CA/CPR. On this scale, normal rats have an NDS of 80, and 0 indicates death.
Statistical analysis. The SPSS 19.0 statistical package (IBM Corp.) was used for statistical analysis. All data were obtained from at least 3 independent experiments. Data are presented as the mean \pm SD. A one-way ANOVA was performed to analyze multiple group comparisons of quantitative data. Bonferroni post hoc test was used after the one-way ANOVA. $\mathrm{P}<0.05$ was considered to indicate a statistically significant difference.

\section{Results}

Characterization of hUSCs. Immunofluorescence staining demonstrated that hUSCs expressed NF200 and GFAP following neurogenesis induction in vitro, which suggested that hUSCs had the potential for multidirectional differentiation (Fig. 2A). The immunophenotypes of hUSCs were determined using flow 


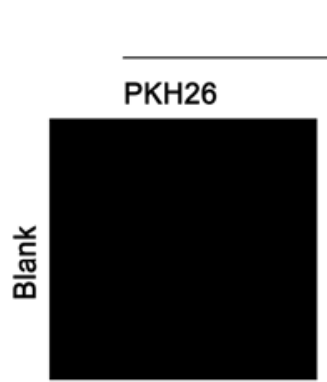

Hippocampus
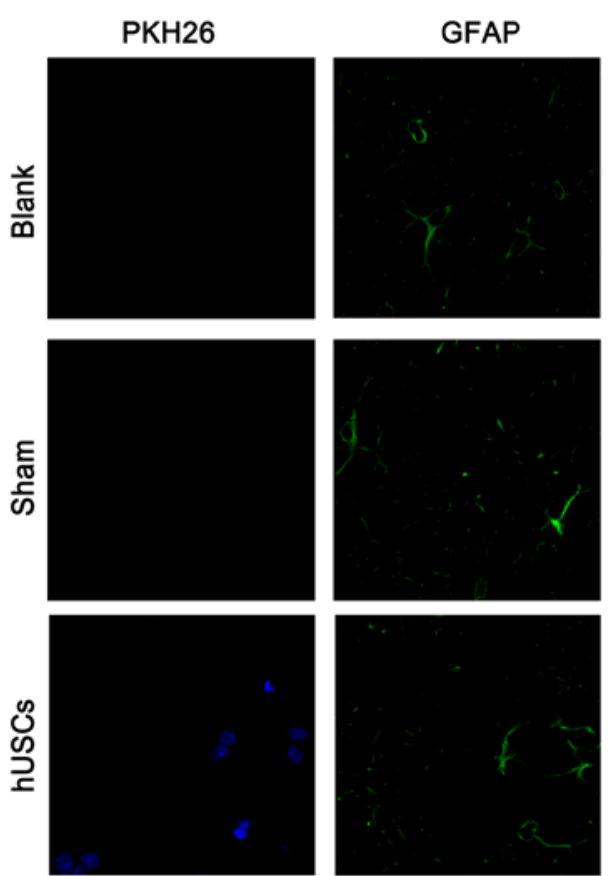
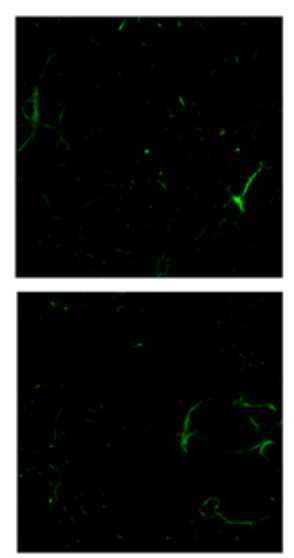
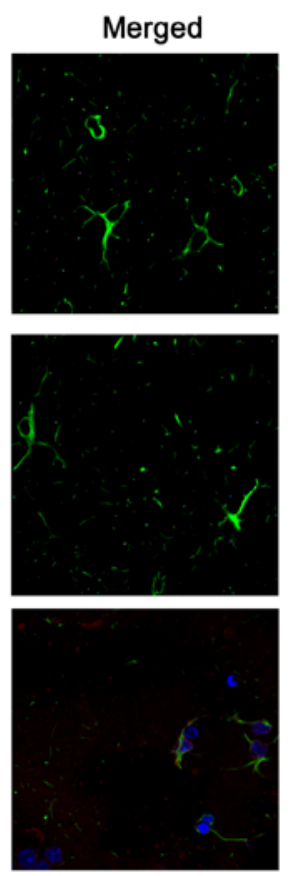

Temporal cortex
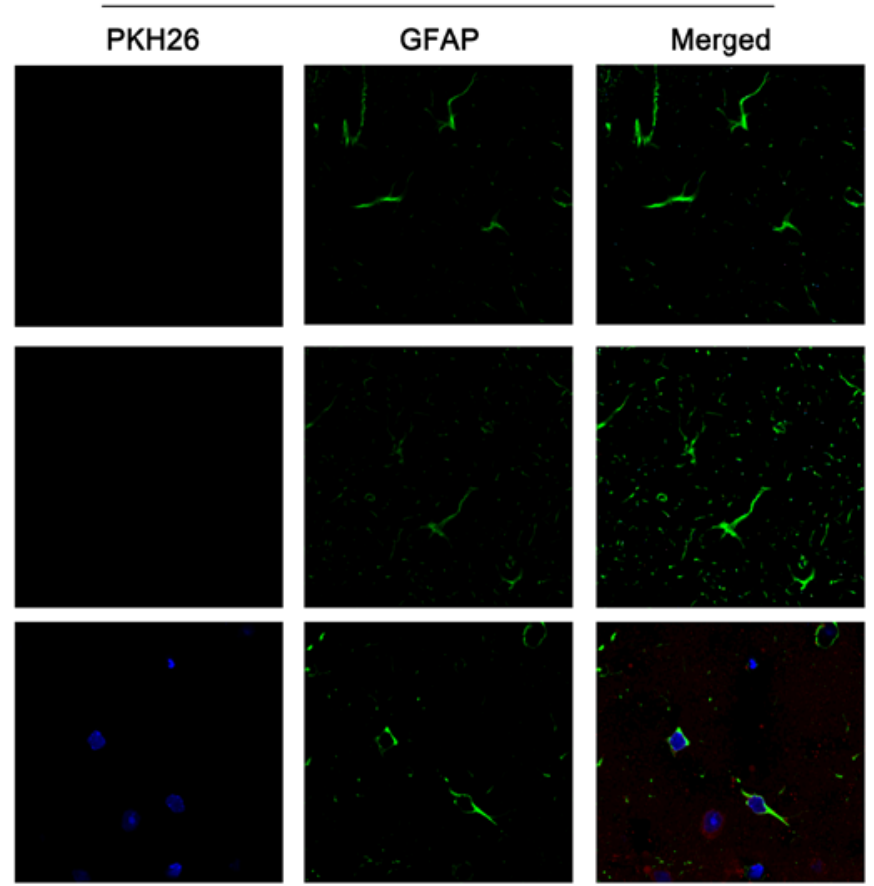

Figure 3. Expression levels of GFAP and PKH26 in the hippocampus and temporal cortex, as evaluated using immunofluorescence. Magnification, x100; scale bar, $50 \mu \mathrm{m}$. High expression of PKH26-labeled hUSCs was observed after hUSC transplantation. GFAP, glial fibrillary acidic protein; hUSC, human urine-derived stem cells.

cytometry. It was found that the surface markers CD29, CD90, CD44, CD105, CD73, CD224, CD146, CD34 and HLA-DR were expressed on 97.6, 84.1, 76.1, 81.7, 96.9, 62.8, 73.5, 0.45 and $0.74 \%$ of hUSCs, respectively (Fig. 2B).

Prognosis of the rats and the distribution of hUSCs after transplantation. In two rats, one from the hUSCs group, one from the blank group and none from the sham group, CPR failed. The remaining rats survived and met the experimental criteria.

To observe the distribution of cells in the brain, hUSCs were labeled with PKH26 before transplantation. High expression of PKH26 was identified in the hippocampus and temporal cortex of rats after pretreatment with hUSCs in comparison with sham group or blank group, indicating that hUSCs aggregated and were distributed in brain tissue following CA/CPR (Fig. 3).

BDNF and VEGF expression levels after hUSC transplantation. To evaluate the expression levels of BDNF and VEGF in brain tissue following hUSC transplantation, an immunofluorescence assay was performed. The results demonstrated that the expression levels of BDNF and VEGF in both the hippocampus and temporal cortex were significantly decreased after CA/CPR. Moreover, pretreatment with hUSC transplantation significantly increased the expression levels of BDNF and VEGF in brain tissue after CA/CPR in comparison with blank group (Fig. 4).

Evaluation of brain tissue injury after hUSC transplantation. To assess the degree of recovery from brain injury after hUSC transplantation, brain water content, serum S100B levels and apoptosis were evaluated. These experiments demonstrated that brain water content and serum S100B levels were significantly increased after $\mathrm{CA} / \mathrm{CPR}$, and that these increases were significantly reversed by hUSC transplantation. In addition, western blot results show that the expression of Bax was augmented, accompanied by the downregulation of Bcl-2 and C-caspase-3, in the hUSC transplantation group compared to the blank group. These data indicate that hUSC pretreatment notably inhibited apoptotic (Fig. 5).

NDS after hUSC transplantation. To examine neurological function after hUSC transplantation, NDS were evaluated. The results suggested that NDS were significantly decreased after $\mathrm{CA} / \mathrm{CPR}$, and pretreatment with hUSCs significantly increased neurological function following CA/CPR (Fig. 6).

\section{Discussion}

Currently, stem cell therapy is considered one of the most promising treatments for numerous refractory diseases, and it has been revealed to exert neuroprotective effects in several models of neurotrauma and degenerative neuropathies $(25,26)$. Intravenous stem cell infusion can effectively improve the prognosis of patients and is associated with the inhibition of apoptosis (27). Previous studies have reported that mesenchymal stem cells (MSCs) can significantly reduce global cerebral ischemia-reperfusion injury (GCIRI), providing a new prospective strategy for brain resuscitation (28). MSCs can be induced to differentiate into neuronal precursor cells or neuron-like cells in vitro and in vivo (29). Animal experiments on focal cerebral ischemia have shown that MSCs can successfully accumulate around the injured brain area, eventually rescuing injured neurons, inducing nerve regeneration and 
A

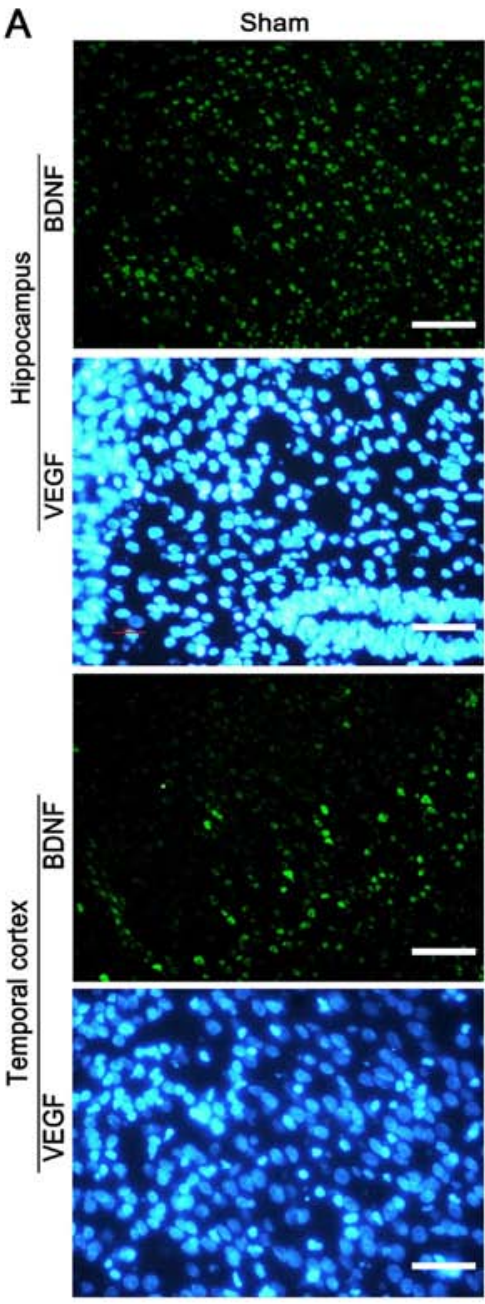

B

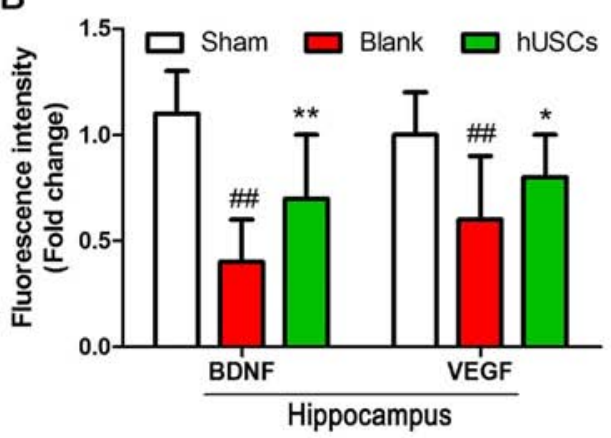

Blank
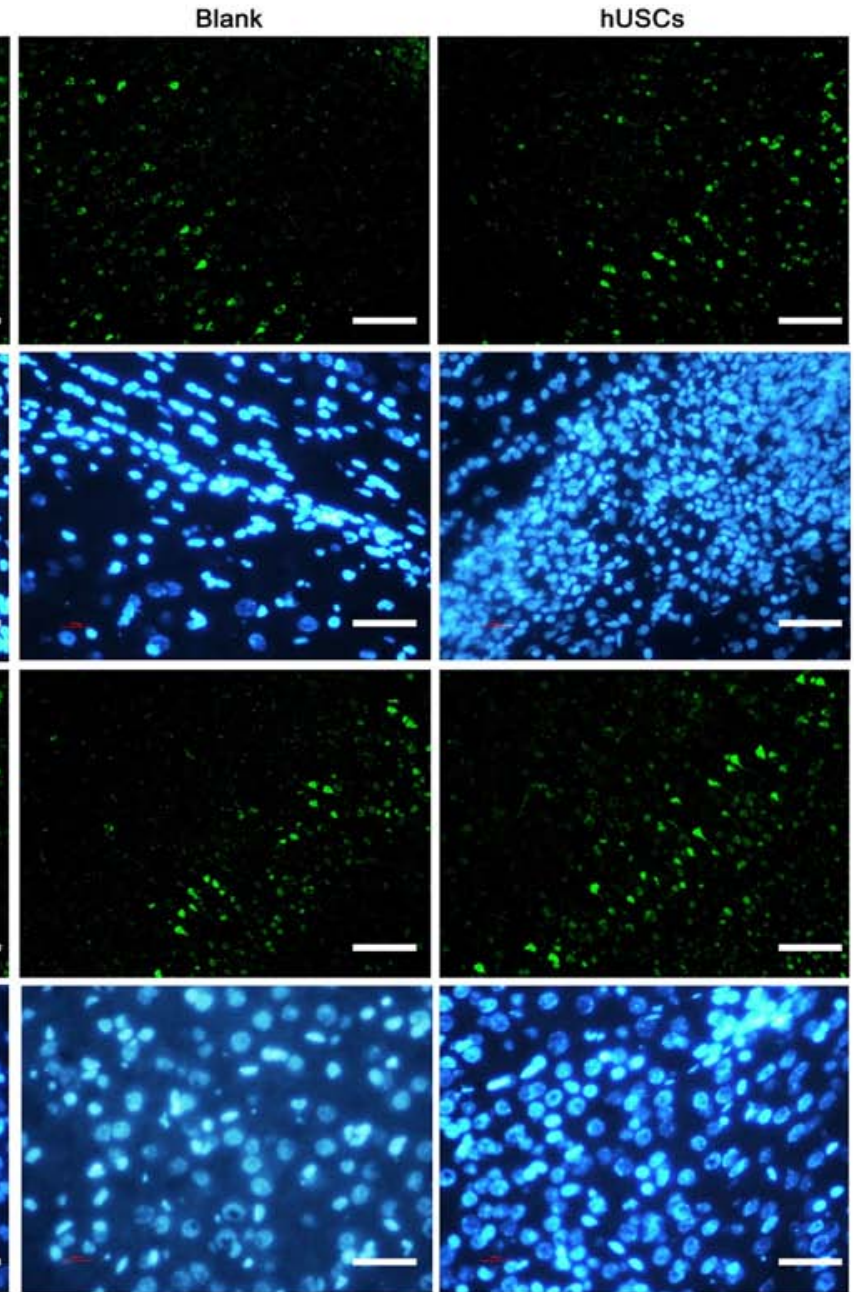

C

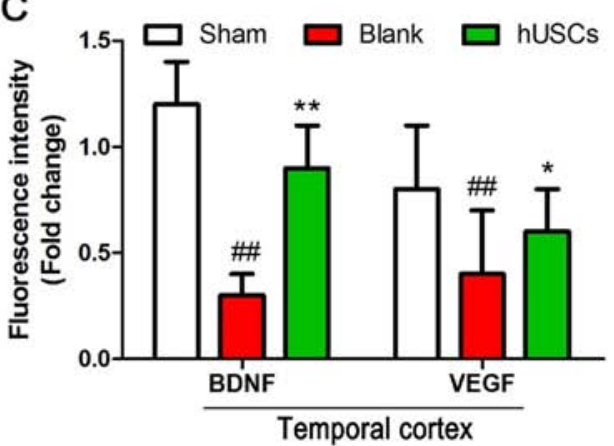

Figure 4. Expression levels of BDNF and VEGF in the hippocampus and temporal cortex, as evaluated using immunofluorescence. (A) BDNF and VEGF immunofluorescence in the hippocampus and temporal cortex. Fluorescence microscope magnification, $\mathrm{x} 40$; scale bar, 200 $\mu \mathrm{m}$. (B) Fold change in BDNF and VEGF expression levels in the hippocampus. (C) Fold change in BDNF and VEGF expression levels in the temporal cortex. ${ }^{\# \#} \mathrm{P}<0.01 \mathrm{vs}$. sham group; ${ }^{*} \mathrm{P}<0.05$ and ${ }^{* *} \mathrm{P}<0.01$ vs. blank group. BDNF, brain-derived neurotrophic factor; VEGF, vascular endothelial growth factor; hUSC, human urine-derived stem cells.

promoting functional recovery after MSC transplantation (30). Moreover, the neuroprotective effects of MSC transplantation can be achieved directly via the secretion of neuroprotective compounds or indirectly via the regulation of immune factors, the promotion of angiogenesis or the activation of the endogenous neural stem cell response (31).

hUSCs have been reported to contribute to the repair of nerve injury. For instance, Zhang et al (32) revealed that hUSCs can be induced to differentiate into neuronal cells, and that this is a feasible and suitable approach for neurological disease modeling. Furthermore, Guan et al (33) observed that hUSCs can differentiate into neuron-like cells in the rat brain, and suggested that hUSCs are a promising cell source for tissue engineering and regenerative medicine. The present study demonstrated that the pretreatment of a rat model of CA/CPR with hUSCs significantly improved neurological function following CA/CPR. Immunofluorescence assays identified that hUSCs aggregated in the hippocampus and temporal cortex of rats, as well as promoted the expression levels of BDNF and VEGF in both areas. Further experiments indicated that brain edema and serum S100B levels were significantly increased after $\mathrm{CA} / \mathrm{CPR}$ and that pretreatment with hUSCs significantly reversed this trend. 

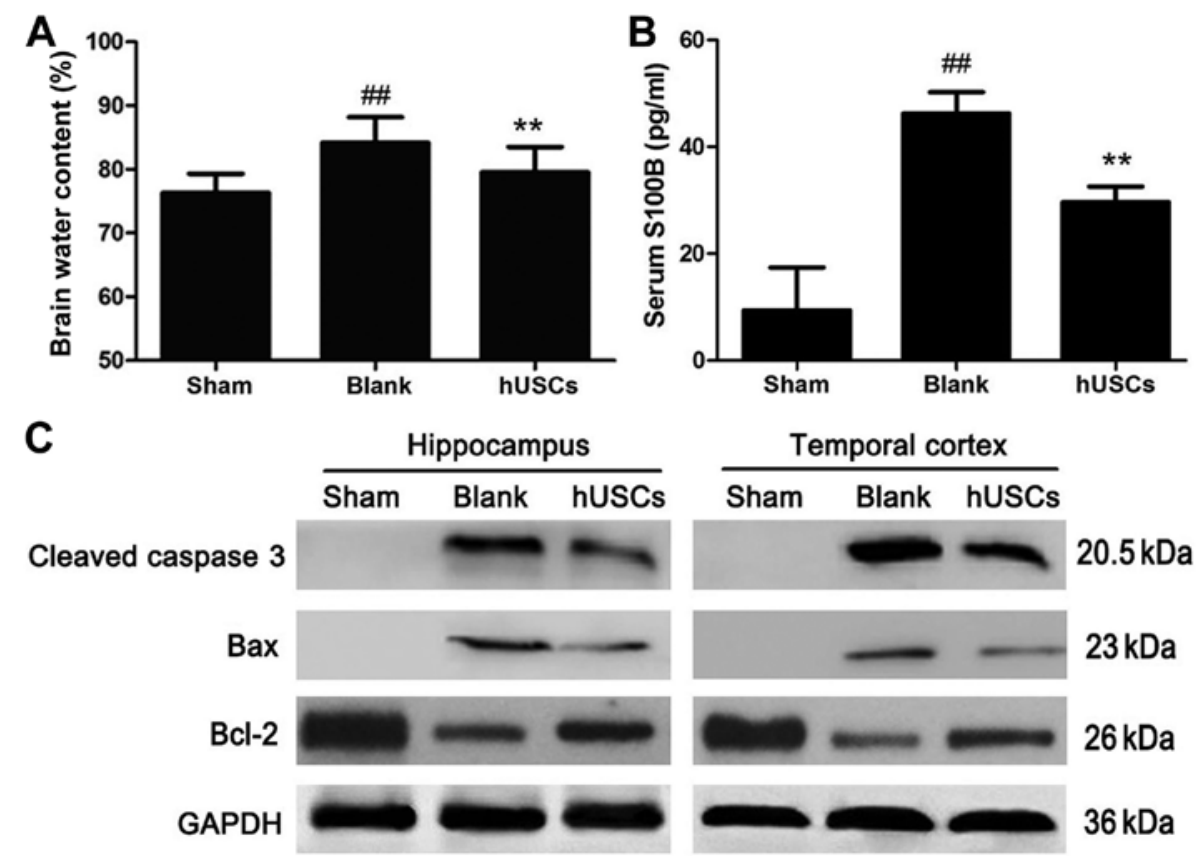

Figure 5. Evaluation of brain tissue injury. (A) Brain water content was detected via a dry-wet assay. (B) Serum S100B levels were determined using an ELISA kit. (C) Apoptotic proteins, such as cleaved caspase 3 and Bax/Bcl-2, were detected using western blotting. ${ }^{\# \#} \mathrm{P}<0.01$ vs. sham group; ${ }^{* *} \mathrm{P}<0.01$ vs. blank group. S100B, S100 calcium binding protein B; hUSC, human urine-derived stem cells.

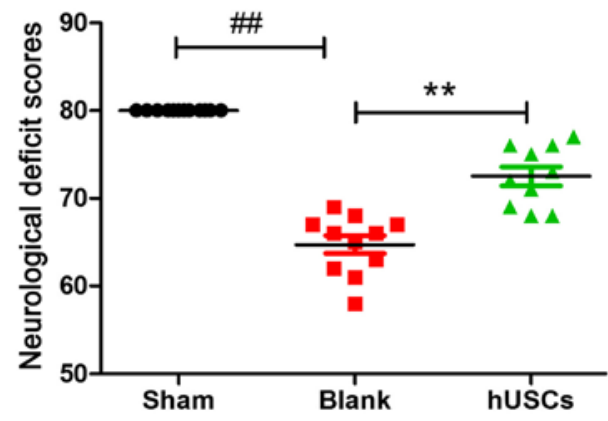

Figure 6. Neurological deficit scores after hUSC transplantation. Neurological function was assessed using neurological deficit scores. ${ }^{\# \#} \mathrm{P}<0.01$ vs. sham group; ${ }^{* *} \mathrm{P}<0.01$ vs. blank group. hUSC, human urine-derived stem cells.

ROS are a class of metabolic substances important for the maintenance of human life. For example, ROS reflect the oxidative stress status of the body, participate in the regulation of human physiology and pathology and maintain the homeostasis of cells (34). Increasing levels of ROS result in damage to DNA, the destruction of the endothelium of tubules and apoptosis (35). To assess the effect of hUSCs on apoptosis in the brain, apoptotic proteins, such as caspase 3 and $\mathrm{Bax} / \mathrm{Bcl}-2$, were detected using western blotting. It was found that hUSC pretreatment notably inhibited neuronal apoptosis in both the hippocampus and temporal cortex following CA/CPR.

Considering the potent neuroprotective properties of hUSCs reported in previous studies $(36,37)$, it was hypothesized that an improved neurological outcome may be achieved via earlier hUSC transplantation after CA/CPR. In the current study, rats received three injections of hUSCs ( $6 \mathrm{~h}$ between injections) 1 day before CA/CPR was established. Brain tissues, especially the hippocampal and temporal cortex tissues, are considered potential targets for drug therapy (38). In the current study,
PKN26-labeled hUSCs were injected via the caudal vein, and it was identified that a sufficient number of hUSCs aggregated in the hippocampus and temporal cortex, suggesting that hUSCs effectively reached brain tissues by passing through the blood-brain barrier (BBB) (39). The present results indicated that hUSCs, similar to MSCs, can cross the BBB and prevent $\mathrm{BBB}$ disruption and endothelial damage, which are initiated in the early phase of GCIRI $(40,41)$. Thus, the current study provides preclinical experimental data for the future clinical application of hUSCs in patients with CA. While the hUSC platform remains in its infancy, this technology is expected to be further developed and adapted by further research. Moreover, future studies should focus on the directed differentiation and efficiency of hUSCs in brain tissues.

There are certain limitations to the current study. First, as immunosuppressant drugs were not used, immune rejection may have occurred in this rat model, and subsequent studies should further analyze and confirm this possibility. Secondly, the directed differentiation and efficiency of hUSCs in brain tissues should be further studied in vivo and in vitro.

In conclusion, to the best of our knowledge, the current study was the first to demonstrate that hUSC transplantation can effectively improve the neurological function of rats following CA/CPR, possibly by promoting the expression levels of BDNF and VEGF and inhibiting brain edema.

\section{Acknowledgements}

Not applicable.

\section{Funding}

This study was supported by grants from the Scientific Research Topics of Jiangsu Provincial Health Commission 
(grant no. H2018027) and the Suzhou Minsheng Science and Technology Project (grant nos. SS2019068 and SYS201730).

\section{Availability of data and materials}

The datasets used and/or analyzed during the current study are available from the corresponding author on reasonable request.

\section{Authors' contributions}

QL conceived and designed the study; CP was the principal experimenter and the author of the manuscript; XZ assisted the experiment and provided data analysis; QC designed the study and revising this manuscript critically for important intellectual content; LW conducted a literature search and interpreted the data. LW, CP and XZ coordinated with the clinical laboratory for sample collection. All authors read and approved the final manuscript.

\section{Ethics approval and consent to participate}

This study was approved by the Ethics Committee of The Affiliated Suzhou Hospital of Nanjing Medical University. All urine donors gave informed written consent before providing urine samples.

\section{Patient consent for publication}

Not applicable.

\section{Competing interests}

The authors declare that they have no competing interests.

\section{References}

1. Reis C, Akyol O, Araujo C, Huang L, Enkhjargal B, Malaguit J, Gospodarev V and Zhang JH: Pathophysiology and the monitoring methods for cardiac arrest associated brain injury. Int J Mol Sci 18 : 129, 2017.

2. Elmer J and Callaway CW: The brain after cardiac arrest. Semin Neurol 37: 19-24, 2017.

3. Sekhon MS and Griesdale DE: Individualized perfusion targets in hypoxic ischemic brain injury after cardiac arrest. Crit Care 21: 259, 2017.

4. Mulder M and Geocadin RG: Will the promise of drug-induced therapeutic hypothermia be fulfilled? Crit Care Med 42: 221-223, 2014.

5. Liakopoulos OJ, Hristov N, Buckberg GD, Triana J, Trummer G and Allen BS: Resuscitation after prolonged cardiac arrest: Effects of cardiopulmonary bypass and sodium-hydrogen exchange inhibition on myocardial and neurological recovery. Eur J Cardiothorac Surg 40: 978-984, 2011.

6. Pasquier M, Hugli O, Paal P, Darocha T, Blancher M, Husby P, Silfvast T, Carron PN and Rousson V: Hypothermia outcome prediction after extracorporeal life support for hypothermic cardiac arrest patients: The HOPE score. Resuscitation 126: 58-64, 2018

7. Metrailler-Mermoud J, Hugli O, Carron PN, Kottmann A, Frochaux V, Zen-Ruffinen G and Pasquier M: Avalanche victims in cardiac arrest are unlikely to survive despite adherence to medical guidelines. Resuscitation 141: 35-43, 2019.

8. Jentzer JC, Clements CM, Wright RS, White RD and Jaffe AS: Improving survival from cardiac arrest: A review of contemporary practice and challenges. Ann Emerg Med 68: 678-689, 2016.
9. Li M, Song W, Ouyang YH, Wu DH, Zhang J, Wang LX and Li J: Clinical evaluation of active abdominal lifting and compression CPR in patients with cardiac arrest. Am J Emerg Med 35: 1892-1894, 2017.

10. Singh SK, Kumar R and Koonwar S: Epidemiology and outcome of pediatric in-hospital cardiopulmonary resuscitation in Northern India. J Pediatr Intensive Care 2: 55-61, 2013.

11. Hackenhaar FS, Medeiros TM, Heemann FM, Behling CS, Putti JS, Mahl CD, Verona C, da Silva AC, Guerra MC, Gonçalves CA, et al: Therapeutic hypothermia reduces oxidative damage and alters antioxidant defenses after cardiac arrest. Oxid Med Cell Longev 2017: 8704352, 2017.

12. Chen L, Li L, Xing F, Peng J, Peng K, Wang Y and Xiang Z: Human urine-derived stem cells: Potential for cell-based therapy of cartilage defects. Stem Cells Int 2018: 4686259, 2018.

13. Zhao T, Luo D, Sun Y, Niu X, Wang Y, Wang C and Jia W: Human urine-derived stem cells play a novel role in the treatment of STZ-induced diabetic mice. J Mol Histol 49: 419-428, 2018.

14. Yang H, Chen B, Deng J, Zhuang G, Wu S, Liu G, Deng C, Yang G, Qiu X, Wei P, et al: Characterization of rabbit urine-derived stem cells for potential application in lower urinary tract tissue regeneration. Cell Tissue Res 374: 303-315, 2018.

15. Li J, Luo H, Dong X, Liu Q, Wu C, Zhang T, Hu X, Zhang Y, Song B and Li L: Therapeutic effect of urine-derived stem cells for protamine/lipopolysaccharide-induced interstitial cystitis in a rat model. Stem Cell Res Ther 8: 107, 2017.

16. Liu G, Wang X, Sun X, Deng C, Atala A and Zhang Y: The effect of urine-derived stem cells expressing VEGF loaded in collagen hydrogels on myogenesis and innervation following after subcutaneous implantation in nude mice. Biomaterials 34: 8617-8629, 2013.

17. Chen CY, Rao SS, Ren L, Hu XK, Tan YJ, Hu Y, Luo J, Liu YW, Yin H, Huang J, et al: Exosomal DMBT1 from human urine-derived stem cells facilitates diabetic wound repair by promoting angiogenesis. Theranostics 8: 1607-1623, 2018.

18. Iwamuro H, Tachibana Y, Ugawa Y, Saito N and Nambu A: Information processing from the motor cortices to the subthalamic nucleus and globus pallidus and their somatotopic organizations revealed electrophysiologically in monkeys. Eur J Neurosci 46: 2684-2701, 2017.

19. Hai K, Chen G, Gou X, Jiang H, Gong D, Cheng Y, Gong C, Li X, Liu Y, Li H, et al: Monoacylglycerol lipase inactivation by using URB602 mitigates myocardial damage in a rat model of cardiac arrest. Crit Care Med 47: e144-e151, 2019.

20. Huang Y, Gao X, Zhou X, Xie B, Zhang Y, Zhu J and Zhu S: Mitophagy in the hippocampus is excessive activated after cardiac arrest and cardiopulmonary resuscitation. Neurochem Res 45: 322-330, 2020.

21. Yang L, Wang J, Deng Y, Gong C, Li Q, Chen Q, Li H, Jiang C, Zhou R, Hai K, et al: Melatonin improves neurological outcomes and preserves hippocampal mitochondrial function in a rat model of cardiac arrest. PLoS One 13: e0207098, 2018.

22. Sun X, Zheng W, Qian C, Wu Q, Hao Y and Lu G: Focal adhesion kinase promotes BMP2-induced osteogenic differentiation of human urinary stem cells via AMPK and Wnt signaling pathways. J Cell Physiol 235: 4954-4964, 2020.

23. Chen Q, Xia R, Zheng W, Zhang L, Li P, Sun X and Shi J: Metronomic paclitaxel improves the efficacy of PD-1 monoclonal antibodies in breast cancer by transforming the tumor immune microenvironment. Am J Transl Res 12: 519-530, 2020.

24. Gong B, Dong Y, He C, Jiang W, Shan Y, Zhou BY and Li W: Intravenous transplants of human adipose-derived stem cell protect the rat brain from ischemia-induced damage. J Stroke Cerebrovasc Dis 28: 595-603, 2019.

25. Mills RJ, Titmarsh DM, Koenig X, Parker BL, Ryall JG, Quaife-Ryan GA, Voges HK, Hodson MP, Ferguson C, Drowley L, et al: Functional screening in human cardiac organoids reveals a metabolic mechanism for cardiomyocyte cell cycle arrest. Proc Natl Acad Sci USA 114: E8372-E8381, 2017.

26. Bao Z, Han Z, Zhang B, Yu Y, Xu Z, Ma W, Ding F, Zhang L, Yu M, Liu S, et al: Arsenic trioxide blocked proliferation and cardiomyocyte differentiation of human induced pluripotent stem cells: Implication in cardiac developmental toxicity. Toxicol Lett 309: 51-58, 2019.

27. Darkazalli A, Vied C, Badger CD and Levenson CW: Human mesenchymal stem cell treatment normalizes cortical gene expression after traumatic brain injury. J Neurotrauma 34: 204-212, 2017. 
28. Ahn JH, Chen BH, Park JH, Shin BN, Lee TK, Cho JH, Lee JC, Park JR, Yang SR, Ryoo S, et al: Early IV-injected human dermis-derived mesenchymal stem cells after transient global cerebral ischemia do not pass through damaged blood-brain barrier. J Tissue Eng Regen Med 12: 1646-1657, 2018.

29. Satija NK, Singh VK, Verma YK, Gupta P, Sharma S, Afrin F, Sharma M, Sharma P, Tripathi RP and Gurudutta GU: Mesenchymal stem cell-based therapy: A new paradigm in regenerative medicine. J Cell Mol Med 13: 4385-4402, 2009.

30. Ko HR, Ahn SY, Chang YS, Hwang I, Yun T, Sung DK, Sung SI, Park WS and Ahn JY: Human UCB-MSCs treatment upon intraventricular hemorrhage contributes to attenuate hippocampal neuron loss and circuit damage through BDNF-CREB signaling. Stem Cell Res Ther 9: 326, 2018.

31. Hawkins KE, Corcelli M, Dowding K, Ranzoni AM, Vlahova F, Hau KL, Hunjan A, Peebles D, Gressens P, Hagberg H, et al: Embryonic stem cell-derived mesenchymal stem cells (MSCs) have a superior neuroprotective capacity over fetal MSCs in the hypoxic-ischemic mouse brain. Stem Cells Transl Med 7: 439-449, 2018

32. Zhang SZ, Ma LX, Qian WJ, Li HF, Wang ZF, Wang HX and Wu ZY: Modeling neurological disease by rapid conversion of human urine cells into functional neurons. Stem Cells Int 2016: 2452985,2016

33. Guan JJ, Niu X, Gong FX, Hu B, Guo SC, Lou YL, Zhang CQ, Deng ZF and Wang Y: Biological characteristics of human-urine-derived stem cells: Potential for cell-based therapy in neurology. Tissue Eng Part A 20: 1794-1806, 2014.

34. Shokoohi M, Olad Saheb Madarek E, Khaki A, Shoorei H, Khaki AA, Soltani M and Ainehchi N: Investigating the effects of onion juice on male fertility factors and pregnancy rate after testicular torsion/detorsion by intrauterine insemination method. Int J Women's Health Reprod Sci 6: 499-505, 2018.
35. Ameli M, Hashemi MS, Moghimian M and Shokoohi M: Protective effect of tadalafil and verapamil on testicular function and oxidative stress after torsion/detorsion in adult male rat. Andrologia 50: e13068, 2018

36. Li G, Xie B, He L, Zhou T, Gao G, Liu S, Pan G, Ge J, Peng F and Zhong X: Generation of retinal organoids with mature rods and cones from urine-derived human induced pluripotent stem cells. Stem Cells Int 2018: 4968658, 2018.

37. Yi H, Xie B, Liu B, Wang X, Xu L, Liu J, Li M, Zhong X and Peng F: Derivation and identification of motor neurons from human urine-derived induced pluripotent stem cells. Stem Cells Int 2018: 3628578, 2018.

38. Wahul AB, Joshi PC, Kumar A and Chakravarty S: Transient global cerebral ischemia differentially affects cortex, striatum and hippocampus in bilateral common carotid arterial occlusion (BCCAo) mouse model. J Chem Neuroanat 92: 1-15, 2018.

39. Hu J, Yu Q, Xie L and Zhu H: Targeting the blood-spinal cord barrier: A therapeutic approach to spinal cord protection against ischemia-reperfusion injury. Life Sci 158: 1-6, 2016.

40. Garbuzova-Davis S, Haller E, Tajiri N, Thomson A, Barretta J, Williams SN, Haim ED, Qin H, Frisina-Deyo A, Abraham JV, et al: Blood-spinal cord barrier alterations in subacute and chronic stages of a rat model of focal cerebral ischemia. J Neuropathol Exp Neurol 75: 673-688, 2016.

41. Lee JY, Lee HE, Kang SR, Choi HY, Ryu JH and Yune TY: Fluoxetine inhibits transient global ischemia-induced hippocampal neuronal death and memory impairment by preventing blood-brain barrier disruption. Neuropharmacology 79: 161-171, 2014.

This work is licensed under a Creative Commons Attribution-NonCommercial-NoDerivatives 4.0 International (CC BY-NC-ND 4.0) License. 